Behavior of numerical error in pore-scale lattice Boltzmann simulations with simple bounce-back rule: Analysis and highly accurate extrapolation

Siarhei Khirevich, and Tadeusz W. Patzek

Citation: Physics of Fluids 30, 093604 (2018); doi: 10.1063/1.5042229

View online: https://doi.org/10.1063/1.5042229

View Table of Contents: http://aip.scitation.org/toc/phf/30/9

Published by the American Institute of Physics 


\title{
Behavior of numerical error in pore-scale lattice Boltzmann simulations with simple bounce-back rule: Analysis and highly accurate extrapolation
}

\author{
Siarhei Khirevich ${ }^{\mathrm{a})}$ and Tadeusz W. Patzek \\ Ali I. Al-Naimi Petroleum Engineering Research Center, King Abdullah University of Science and Technology, \\ Thuwal, Saudi Arabia
}

(Received 30 May 2018; accepted 28 August 2018; published online 14 September 2018)

\begin{abstract}
We perform the viscosity-independent Stokes flow simulations in regular sphere packings using the two-relaxation-times (TRT) lattice Boltzmann method (LBM) with the simple bounce-back (BB) rule. Our special discretization procedure reduces the scatter in integral quantities, such as drag force, and quantifies the solution convergence error. We assume transition to linear $(-1)$ convergence rate for different sets of TRT parameters and use this assumption to provide a simple extrapolation scheme. After establishing the accurate reference values of drag for a wide range of porosities, $0.26-0.78$, we show a ten-fold decrease in the drag error using the suggested extrapolations. This error decrease allows the simple LBM/BB scheme to reach an accuracy of the high-order interpolated boundary schemes. The suggested extrapolation approach is straightforward to apply in porous media, whose pore space can be discretized at several resolutions. Published by AIP Publishing. https://doi.org/10.1063/1.5042229
\end{abstract}

\section{INTRODUCTION}

Over the last few decades, the lattice-Boltzmann method (LBM) has been widely used to calculate the single- and two-phase flow of incompressible and immiscible fluids ${ }^{1-4}$ in the morphologically and topologically complex pore space of clastics and carbonates. In addition to the accuracy of LBM discretizations, the pore space itself can never be described accurately ${ }^{5-8}$ by the finite resolution reconstructions. This confluence of difficulties with numerical and imaging approximations leads to an interesting question: What fraction of computational inaccuracy in flow and transport calculations is caused by the pore space discretization and how much of it originates in the flow calculations with LBM? In this paper, we try to provide quantitative answers pertinent to the latter.

By extending an earlier result,${ }^{9}$ we derive a simple method of increasing the accuracy of one of the simplest LBM implementations. The key reasons for LBM's popularity in calculating incompressible flows in porous media have been (i) relative simplicity of its implementation and (ii) acceptable accuracy while using regular meshes to resolve complex solid boundaries. The kinetic origin of LBM assumes the use of collision rules for evolving its particles; one of the popular set of rules is the Bhatnagar-Gross-Krook (BGK) collision operator. ${ }^{10-12}$ Despite its popularity, BGK is well known for the dependency of permeability (or drag force) on the viscosity of simulated fluid. ${ }^{13-15}$ Reformulation of the matrix-collision model ${ }^{16}$ resulted in the multiple-relaxation-times (MRT) collision operator with several adjustable parameters. A reduced version of MRT developed later, the two-relaxation-times (TRT) collision operator, ${ }^{17,18}$ is well suited for solving advection-diffusion problems and performing Navier-Stokes simulations. ${ }^{19}$

Until now, choices of relaxation parameters in the TRT and MRT schemes have remained an open question. ${ }^{19}$

a)Electronic mail: siarhei.khirevich@kaust.edu.sa
Analytical evaluation of the TRT operator demonstrated that a specific combination $\Lambda$ (known as the "magic number" 19 ) of its two relaxation parameters controls the viscosity-independent steady solutions in any geometry. In Poiseuille flow simulations using the bounce-back (BB) rule (see below) with walls located midway, the intersected links, $\Lambda=3 / 16$ and $\Lambda=3 / 8$, provide an exact velocity profile in a channel oriented horizontally or diagonally with respect to the lattice; ${ }^{13}$ the value of $\Lambda=1 / 8$ gives the exact average flow rate through the channel. For flows in complex geometries, these values of $\Lambda$ provide solutions of acceptable accuracy for medium resolutions and above, e.g., Ref. 9. It was established ${ }^{9,20}$ that values of $\Lambda$ outside of the interval $[1 / 8,3 / 8]$ can change significantly the simulated flow field in porous media. Therefore, the parameter $\Lambda$ can be a powerful tool that controls simulation accuracy.

Regular sphere packings represent a good underlying geometry to assess the accuracy of LBM simulations in porous media: they are simple enough to be approached (semi-) analytically, yielding a number of independently obtained solutions. ${ }^{21-23}$ At the same time, the converging/divergening flow in packings makes this geometry clearly more complex than flow between two parallel plates or through a square duct. It is also worth mentioning that sphere packings represent real systems in chromatography ${ }^{24}$ and chemical engineering. ${ }^{25}$

Popularity of the lattice Boltzmann method ${ }^{26-29}$ as a flow solver can be attributed to its acceptable accuracy while using the bounce-back (BB) boundary condition, ${ }^{9,30}$ for which the lattice populations facing a solid voxel are reflected back from it. Simplicity, locality, and robustness of BB made it attractive among other boundary rules. However, a detailed analysis of numerical LBM/BB solutions did not prove their convergence to accurate solutions in complex geometries, ${ }^{14,15,30,31}$ despite theoretical analysis suggesting that this must be the case. ${ }^{32}$ Inaccuracies of LBM/BB simulations have led to various improvements, such as an effective hydrodynamic radius ${ }^{33}$ 
or the extrapolation of simulation results toward the infinitely high discretization resolution. ${ }^{34}$

Validation of flow simulations in regular packings against independent solutions is often difficult because of the significant scatter of LBM/BB results. This scatter leads to statements about the different convergence behavior of random and regular packings or to questioning the convergence of LBM/BB to the true solution. ${ }^{30}$ In addition, the ubiquitous use of the -2 convergence rate for extrapolation ${ }^{34,35}$ complicates the understanding of the convergence of LBM/BB in complex geometries.

The interpolated or multi-reflection (IMR) boundary condition algorithms solve for the intersections of the analytical boundary and LBM lattice links. This feature makes LBM/IMR significantly more accurate than LBM/BB. Solutions obtained with the IMR boundary conditions demonstrate much weaker dependency on $\Lambda$ compared with the bounceback rule. ${ }^{9,15}$ Despite improved accuracy and convergence rates, $, 14,30,31,36$ the implementation of LBM/IMR requires additional programming to modify a flow solver ${ }^{30}$ for each type of considered geometries, e.g., for sphere packings vs. fractures. It has been reported that (i) the interpolated boundary conditions do not always conserve the total fluid mass ${ }^{14,36}$ and (ii) some of the advanced boundary condition schemes require more-than-first lattice neighbor, thus diminishing LBM locality and degrading the scaling efficiency of parallel LBM implementations. ${ }^{37}$

In this work, we focus only on the bounce-back boundary condition and extend the results of an earlier study by Khirevich et al. ${ }^{9}$ Using the previously introduced discretization procedure, we reduce significantly the scatter of the solutions. With less scatter, we analyze the behavior of the numerical error and observe convergence to the -1 rate for a wide range of TRT parameters. We suggest a simple extrapolation approach and demonstrate a ten-fold reduction of the relative error in integral quantities such as the permeability or drag.

\section{SIMULATION APPROACH}

We solve the Stokes problem in a periodic domain for the momentum $\vec{j}$ of an incompressible fluid driven by a body force $\vec{B}$,

$$
\begin{gathered}
\mu \Delta \vec{j}+\vec{B}=0, \\
\vec{j}=\rho_{0} \vec{u},
\end{gathered}
$$

where $\mu$ is the kinematic viscosity, $\rho_{0}$ is the fluid density, and $\vec{u}$ is the fluid velocity. The Stokes flow regime is typical of the porous media in which inertia is dominated by viscosity.

The average normalized drag force $F_{\mathrm{d}}$ exerted on a single sphere (cf. van der Hoef et $_{\text {al. }}{ }^{34}$ ) is

$$
F_{\mathrm{d}}=\frac{d_{\mathrm{sp}}^{2}}{18(1-\varepsilon) k},
$$

where $d_{\mathrm{sp}}$ is the sphere diameter, $\varepsilon$ is the porosity, and $k$ is the permeability. Later in this work, we consider $d_{\text {sp }}$ to be expressed in lattice units and $\varepsilon$ is taken as the ratio of $f l u i d$ voxels to the total number of voxels. Permeability is calculated using Darcy's law,

$$
k \equiv k_{x}=\frac{\mu \varepsilon j_{x}}{B_{x}} .
$$

In the equation above, we assume that the simulation domain is isotropic and the permeability tensor is a scalar.

\section{A. Two-relaxation-times lattice Boltzmann scheme}

To solve Eq. (1), we employ the two-relaxation-times lattice Boltzmann method. ${ }^{17-20,38}$ LBM simulates the behavior of fictitious particles that reside on a discrete cubic lattice and evolve at discrete time steps $t$. Each lattice site $\vec{x}$ is occupied by $Q$ (=19 in this study) particle populations $f_{q}$, consisting of one immobile particle and the $Q_{m}=Q-1$ mobile ones. The lattice sites are interconnected by $Q_{m}$ velocity vectors $c_{q}$. Using a symmetry argument, we group the velocity vectors into "links" $\left\{c_{q}, c_{\bar{q}}\right\}$ such that $c_{\bar{q}}=-c_{q}$. In each time step, the particles propagate along the lattice vectors and collide according to the following rule:

$$
f_{q}\left(\vec{x}+\vec{c}_{q}, t+1\right)=\tilde{f}_{q}(\vec{x}, t),
$$

$$
\begin{gathered}
\tilde{f}_{q}(\vec{x}, t)=\left[f_{q}-s^{+}\left(f_{q}^{+}-e_{q}^{+}\right)-s^{-}\left(f_{q}^{-}-e_{q}^{-}\right)+S_{q}^{+}+S_{q}^{-}\right](\vec{x}, t), \\
q=0, \ldots, Q_{m} / 2, \\
\tilde{f}_{\bar{q}}(\vec{x}, t)=\left[f_{\bar{q}}-s^{+}\left(f_{q}^{+}-e_{q}^{+}\right)+s^{-}\left(f_{q}^{-}-e_{q}^{-}\right)+S_{q}^{+}-S_{q}^{-}\right](\vec{x}, t), \\
q=1, \ldots, Q_{m} / 2,
\end{gathered}
$$

where $f_{q}^{ \pm}=\left(f_{q} \pm f_{\bar{q}}\right) / 2$, tilde $\sim$ denotes the post-collision state, and the equilibrium functions $e_{0}$ and $e_{q}^{ \pm}$are calculated using the local mass $\rho$ and momentum $\vec{J}$ as

$$
\begin{gathered}
\rho=f_{0}+2 \sum_{q=1}^{Q_{m} / 2} f_{q}^{+}, \quad \vec{j}=\vec{J}+\frac{1}{2} \vec{B}, \quad \vec{J}=2 \sum_{q=1}^{Q_{m} / 2} f_{q}^{-} \vec{c}_{q}, \\
e_{q}^{+}=c_{s}^{2} t_{q} \rho, \quad e_{q}^{-}=t_{q}\left(\vec{J} \cdot \vec{c}_{q}\right), \quad \text { and } \quad e_{0}=\rho-2 \sum_{q=1}^{Q_{m} / 2} e_{q}^{+} .
\end{gathered}
$$

Here $c_{s}^{2}$ denotes the speed of sound and $t_{q}$ are the isotropic weights of $1 / 6$ and $1 / 12$ for the 6 "horizontal" and 12 "diagonal" lattice vectors, respectively. The quantity $S^{+}$denotes the mass sources, here equal 0 , while $S^{-}$is the body force driving the fluid,

$$
S_{q}^{-}=t_{q} \rho_{0}\left(\vec{B} \cdot \vec{c}_{q}\right) .
$$

The relaxation parameters $s^{ \pm}$are limited to the ]0, 2[ interval, and a particular value of $s^{+}$defines the fluid viscosity as $v=\left(1 / s^{+}-1 / 2\right) / 3$, while the parameter $s^{-}$is free-tunable. Let us introduce the following ratio between $s^{+}$and $s^{-}$:

$$
\Lambda=\left(1 / s^{+}-1 / 2\right)\left(1 / s^{-}-1 / 2\right),
$$

which is also known as the "magic number". ${ }^{19}$ The ratio $\Lambda$ controls the location of the zero-velocity boundary within the simulated geometry (for instance, see Fig. 1 in Ref. 9) and therefore defines the stationary flow field up to machine accuracy for any combination of the two relaxation parameters $s^{ \pm}$ as well as body force values. ${ }^{17,19}$ In other words, $\Lambda$ directly controls the output integral quantities such as total momentum, permeability, and drag force. 
All the results in this paper can be reproduced using the BGK collision operator, keeping in mind the following relations between $\Lambda$, fluid viscosity, and the single relaxation parameter $\tau$ of the BGK model:

$$
\begin{aligned}
& \Lambda=9 v^{2}, \\
& \Lambda=\left(\tau-\frac{1}{2}\right)^{2} .
\end{aligned}
$$

In the BGK collision operator, the fluid viscosity is controlled solely by $\tau$. Therefore, selecting $\tau$ at a value that matches the TRT solution with a given $\Lambda$ fixes the corresponding value of $v$. As a result, a non-optimal combination of the viscosity $v$, desired accuracy, and simulation geometry may negatively impact execution time to reach the steady state (see Fig. 5 in Ref. 20 and Fig. 7 in Ref. 9).

\section{B. Steady-state solution}

Evolution of LBM solution in pore-scale simulations typically demonstrates oscillatory behavior (for example, see Fig. 7 in Ref. 9), which mainly depends on the lattice viscosity as well as on the geometry of the simulation domain. In this work, our goal is to estimate integral quantities, such as drag force, and therefore we use the following termination criterion:

$$
M_{T}^{i}=\left|\frac{j_{x}^{i}-j_{x}^{i-1}}{j_{x}^{i}}\right|<\delta, \quad \text { for } \quad i=i, \ldots, i-T,
$$

which is based on the momentum $j_{x}$ calculated along the direction of the applied body force $B_{x}>0$. This criterion can be understood as follows: simulation is terminated when the relative variation of momentum $j_{x}$ between any pair of two consecutive iterations $i-1$ and $i$ does not exceed $\delta$ over $T$ previous iterations. We stress the importance of satisfying the termination rule (13). Otherwise, the evaluation of $M_{1}^{i}$ for a single iteration pair $(i-1, i)$, even with a very small $\delta$ may terminate simulation too early. This can occur at the time of development of another momentum oscillation, followed by a slow but noticeable change in $j_{x}$. Our results are obtained for $\varepsilon=10^{-9}$ and $T=200$, while the fluid viscosity is $v=0.5$ (or $\left.s^{+}=0.5\right)$.

\section{Discretization}

The quality of mapping of analytical boundaries onto a discrete lattice is crucial to the accuracy of simulations in regular geometries such as periodic arrays of spheres. The main problem is that by default, (i) each periodic cell contains just a few geometrical elements (spheres) to be discretized and (ii) the discrete lattice must contain an integer number of nodes along each dimension. In practice, substantial variation in lattice geometry can occur for a slight change of discretization resolution. ${ }^{9}$ We note that this problem was partially addressed by Chun and Ladd, see Fig. 8 in Ref. 36, who randomly moved the unit cell with spheres relative to the lattice and analyzed the corresponding error behavior. In the case of irregular sphere packings, this problem vanishes when the number of spheres becomes sufficiently large to sample the underlying lattice in different ways.

In this work, we apply the discretization approach that was suggested previously, see Fig. 5 in Ref. 9 and the supporting explanations. This approach exploits the periodicity property of a regular geometry, according to which we replicate a unit cell $U$ times along each Cartesian direction and take the underlying lattice dimension $L$ not evenly divisible by $U$. This leads to the non-integer lattice size per unit cell $L_{\text {unit }}$, while having integer $L$ for the whole lattice. In practice, the use of non-integer values of $L_{\text {unit }}$ significantly reduces scatter in the simulated drag force (permeability), depicted in Fig. 1. Extending the arguments above, the suggested discretization approach gives the best results when $L$ and $U$ have no common integer multipliers. For example, taking $L=30$ and $U=4$ ( $L \bmod U \neq 0$ ) results in a drag value equivalent to the simulation setup with $L=15$ and $U=2$ because $L=30$ and $U=4$ have a common multiplier of 2 ; a better choice for $U=4$ and $L \approx 30$ is $L=29$ or $L=31$.

A particular choice of $U$ depends on the resolution and the porosity. From our experience of dealing with the bodycentered cubic (BCC), face-centered cubic (FCC), and simple cubic (SC) packings of touching spheres, very low resolutions of $0.1-1$ of lattice nodes per sphere diameter can require as many as $U \approx 10$ (resulting in $U^{3}$ unit cells), while for high resolutions of several hundred lattice nodes, $U=2$ may be

\section{1 unit cell (standard)}

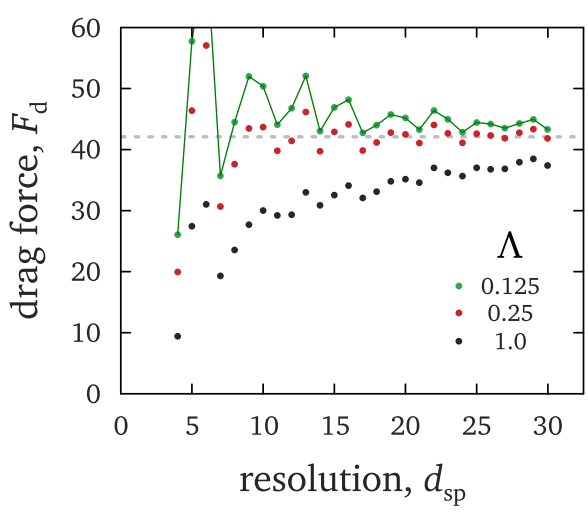

$2^{3}$ unit cells

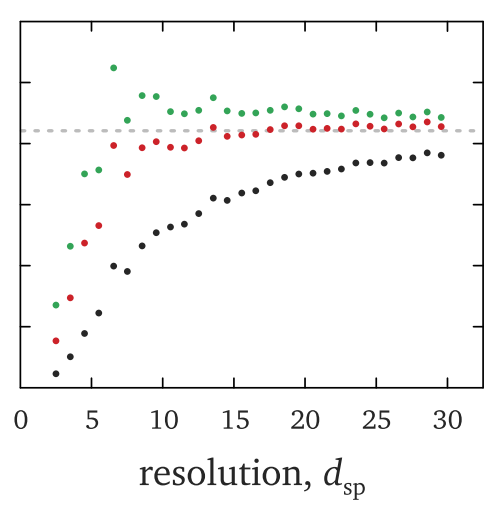

$5^{3}$ unit cells

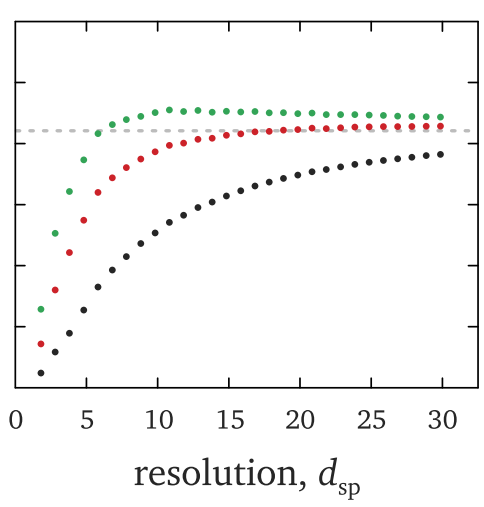

FIG. 1. Drag force vs. discretization resolution (in lattice units per sphere diameter) for a simple cubic packing of touching spheres with $\varepsilon \approx 0.476$. Green, red, and black colors denote $\Lambda=0.125,0.25$, and 1.0, respectively. The reference drag value for this geometry is 42.1 (dashed line). 
sufficient. It appears that the number of lattice voxels crossed by spheres in different ways is the key parameter that reduces scatter.

Our discretization approach produces the fully symmetric discrete lattices, i.e., each discretized geometry has reflection symmetry in all three Cartesian directions. In addition, we perform a slight variation of the sphere radius $d_{s p}$ around its analytical value in order to obtain better agreement with the analytical and discrete porosities. The corresponding MATLAB scripts are available online. ${ }^{39}$

\section{Considered geometries and reference drag values}

In this paper, we deal solely with regular packings of spheres that are commonly used to validate the numerical Stokes solutions $9,15,30,36,40-42$ and enable the in-depth comparisons of numerical simulations with previous studies. This type of geometry can be approached analytically, ${ }^{21-23}$ but at the same time, it captures the key features of flow in porous media and demonstrates more complex flow patterns, relative to open channels or pipes with circular or quadratic cross sections. We note that our approach is not limited to regular sphere packings and is applicable to the complex geometries, where the discretization resolution can be varied.

We consider three basic packing types (Fig. 2) with unit cells of cubic shape and assume that they are filled with spheres of unit diameter. Face-centered cubic (FCC) packing has a unit cell edge $\sqrt{2}$ long and is composed of 14 spheres: 6 are located in the centers of each face while 8 spheres are in the corners of the FCC unit cell. Body-centered cubic (BCC) packing has one sphere in the cell center and 8 in the corners, and its edge is $2 \sqrt{3}$ long. Simple cubic (SC) packing has unit edge length and a single sphere in the cell center. For all three geometries,

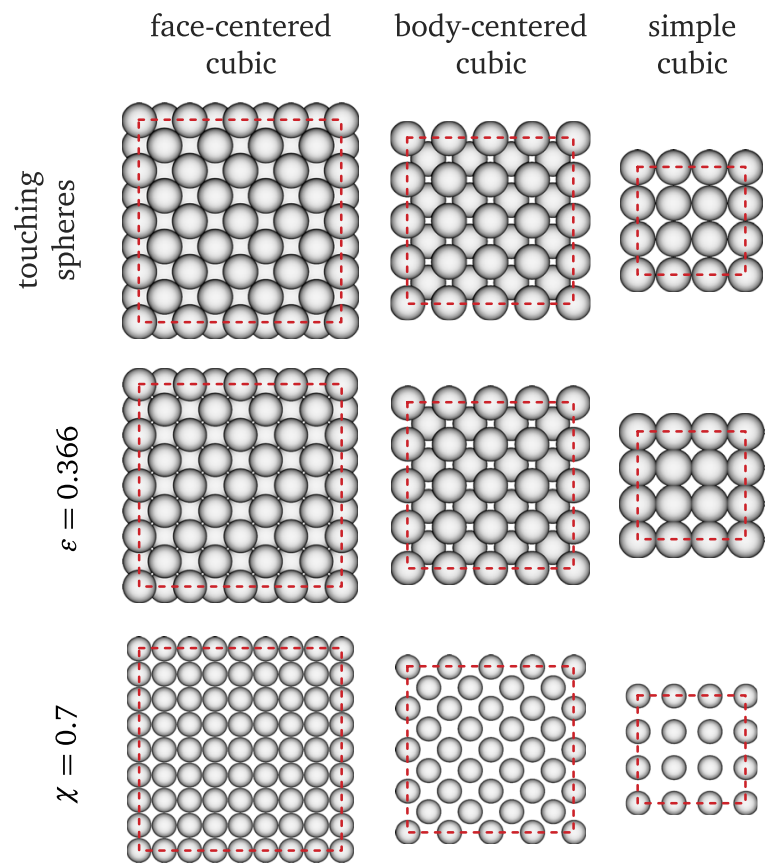

FIG. 2. Three packing types used in the simulations with unit cells replicated 4 times along each direction; the actual simulation domain is indicated with the red dashed lines. The middle row shows packings of fixed porosity ( $\varepsilon=0.366$ ), while the top and bottom rows depict touching and diluted packings, respectively.
TABLE I. Reference drag values used in this work; packing types and porosities are given in the left column and top row, respectively. The value of $\chi=0.7$ is the diameter scaling factor, which equals to 1.0 when spheres are touching. The factor $\chi$ is related to porosity as $\varepsilon=1-\chi^{3}\left(1-\varepsilon^{*}\right)$, with $\varepsilon^{*}$ denoting the porosity of a packing with touching spheres. Porosity values in bold are approximate and calculated as $\varepsilon_{\mathrm{fcc}}^{*}=1-\pi / \sqrt{18} \approx 0.26$, $\varepsilon_{\mathrm{bcc}}^{*}=1-\pi \sqrt{3} / 8 \approx 0.32$, and $\varepsilon_{\mathrm{sc}}^{*}=1-\pi / 6 \approx 0.48$. Drag values in normal font are taken from the LBM/MCLI simulations ${ }^{9}$ while bold values are from the 30-term series expansion by Sangani and Acrivos. ${ }^{21}$ The underlined drag value for the SC packing with the overlapping spheres and porosity of 0.366 is estimated from the extrapolation approach presented in this work with $d_{\mathrm{sp}}^{\max } \approx 429$ und $U=3$.

\begin{tabular}{lcccccc}
\hline \hline & $\mathbf{0 . 2 6}$ & $\mathbf{0 . 3 2}$ & 0.366 & $\mathbf{0 . 4 8}$ & $\chi=0.7$ & 0.784 \\
\hline FCC & 432.0 & & 146.5 & & $\mathbf{9 . 7 1 6 5}$ & $\mathbf{7 . 7 5 7 6}$ \\
BCC & & 162.8 & 114.4 & & $\mathbf{8 . 5 6 3 1}$ & $\mathbf{7 . 7 3 8 8}$ \\
SC & & & $\underline{84.85}$ & 42.10 & $\mathbf{6 . 0 0 4 2}$ & $\mathbf{7 . 4 4 3 2}$ \\
\hline \hline
\end{tabular}

only parts of spheres that are inside of the corresponding unit cell are considered in flow simulations.

In this study, we deal with accuracies of $10^{-3}$ or better in estimating drag force values. Special attention is given to the reference drag values because they should be trusted with the accuracy of at least $10^{-4}$. A careful examination of the previous non-LBM approaches for estimating drag in packings of the touching SC, BCC, and FCC spheres reveals disagreement between the studies by Zick and Homsy, ${ }^{22}$ Sangani and Acrivos, ${ }^{21}$ and Larson and Higdon ${ }^{23}$ (see Table 5 in Ref. 9). Therefore, for touching spheres, we took drag values obtained from the high-resolution LBM simulations with the "parabolic" multi-reflection (MCLI) boundary condition. For the MCLI boundary condition, (i) there is almost no dependency on $\Lambda$ and (ii) the fast convergence rates of -3 or -4 provide the practically resolution-independent values of drag at high resolutions, with $>100$ lattice nodes per sphere diameter.

Reducing sphere diameters with the contraction factor $\chi<1$ increases the distance between sphere surfaces and results in better agreement among the published studies. ${ }^{21-23}$ Specifically, at the accuracy of at least $10^{-4}$, the literature drag values become identical when $\chi \approx 0.7$ or less. Sangani and Acrivos $^{21}$ provided a series expansion for the estimation of drag in FCC, BCC, and SC packings, which we adopt here to complement our study with the accurate drag force values in regular packings of high porosity. The corresponding values for the three regular packing types are listed in Table I.

The set of reference drag values for the touching and diluted regular packings is extended to the porosity of 0.366 . For this porosity, there are solutions for BCC and FCC geometries available from the work of Khirevich et al. ${ }^{9}$ These solutions were obtained at high-resolutions using LBM with the "parabolic" multi-reflection boundary schemes. ${ }^{14}$ We also support this porosity with the SC geometry of overlapping spheres. We estimate its drag force with the approach presented in this paper.

\section{GENERAL BEHAVIOR OF NUMERICAL ERROR}

The two-relaxation-times collision operator controls the steady-state drag force solely through $\Lambda$. The $\Lambda$-dependency 

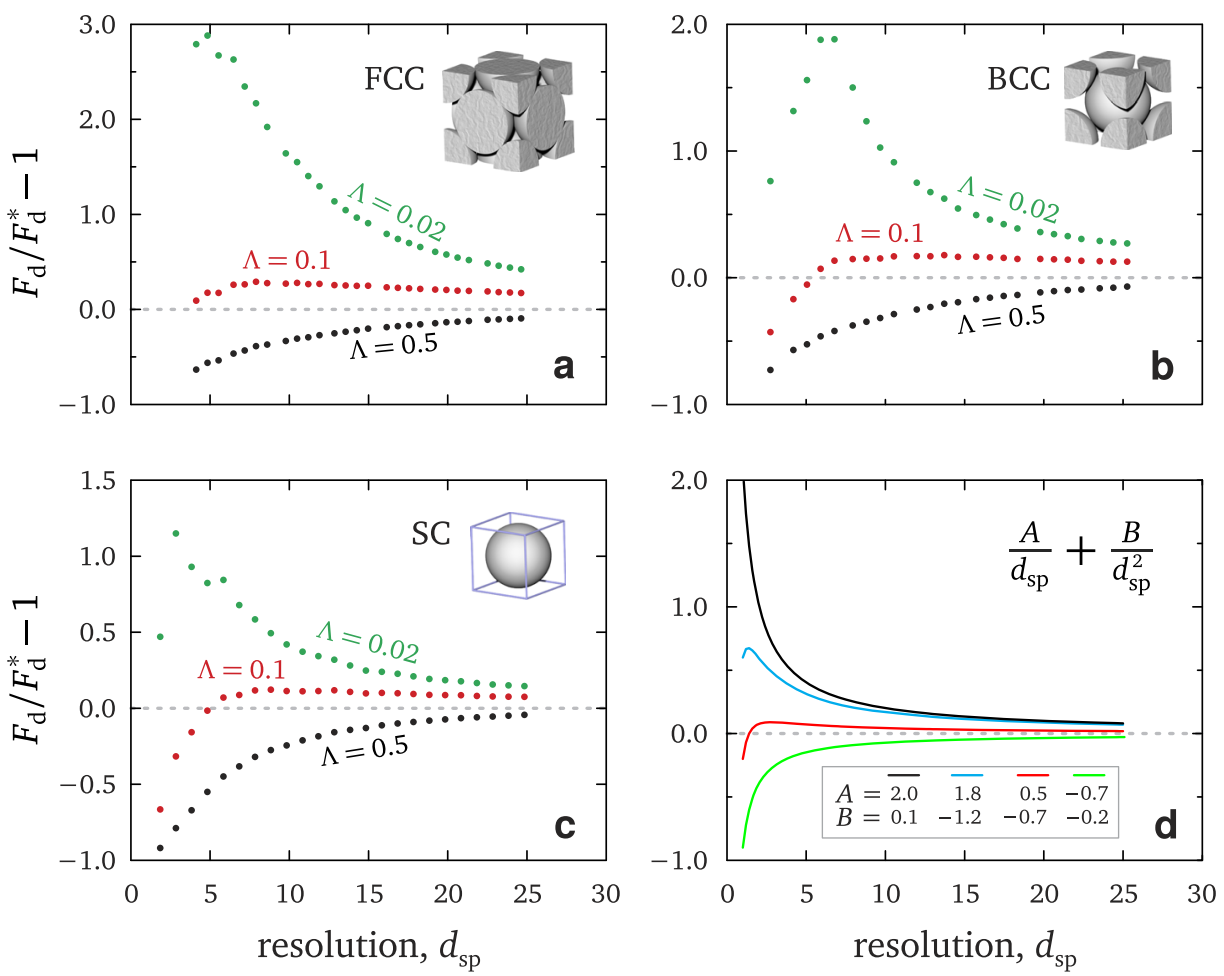

FIG. 3. [(a)-(c)] Relative drag force error as a function of discretization resolution for FCC, BCC, SC packings of touching spheres; the dependencies are shown for three $\Lambda$ values. (d) A simple model that mimics the variation of the quadratic and linear error convergence rates with resolution; shown are four different combinations of model parameters $A$ and $B$. of the numerical solutions obtained with the bounce-back scheme in three regular packings is demonstrated in Fig. 3. As mentioned previously, ${ }^{9}$

- $\Lambda$ has the strongest impact on the numerical error at low resolutions;

- the error is non-monotonic with resolution increase, and it can significantly over- or underestimate true solution depending on a particular value of $\Lambda$; and

- error behavior is similar for random and regular geometries or different porosities meaning that it is related to the method itself rather than to a particular type of porous media.

In Fig. 3, we further extend the understanding of error behavior. The non-monotonic convergence in Figs. 3(a)-3(c) can be related to the interplay among different error contributions: away from solid boundaries, the lattice Boltzmann method performs with second-order accuracy, ${ }^{32}$ while the first order ${ }^{13}$ bounce-back boundary condition is applied at the boundary nodes. This suggests the existence of at least two contributions to the total simulation error, with quadratic and linear behavior. (There is one more contribution, related to the voxel-wise integration of three-dimensional flow field when calculating integral quantities such as average flow rate, but we omit it here.) An important point is that these contributions can have different signs and therefore compensate each other. This idea was discussed earlier, for instance, in relation to the simulations of hypersonic flows. ${ }^{43}$ Mutual compensation of error contributions can lead to the very slow transient convergence rates, such as $-0.2 \ldots-0.4$. Very slow convergence can be seen in other studies with the standard choice of relaxation parameters equivalent to $\Lambda=0.25$ ( $\tau=1$ in the BGK collision operator). ${ }^{30,40,41}$
The bulk and boundary error contributions could eliminate each other, resulting in zero total error, but this never happens over the continuously varying resolutions because convergence rates of the two contributions are different. In Fig. 3(d), we provide a simple expression, $A / d_{\mathrm{sp}}+B / d_{\mathrm{sp}}^{2}$, which captures most of the error behavior obtained from the simulations. In fact, $\Lambda$ can be seen as a parameter controlling the relative impact and sign of each error contribution.

Figure 4 is a double logarithmic plot of the low and high porosity cases in order to analyze their convergence rates. Here we consider (a) the diluted FCC and BCC packings as well as the condensed SC packing of overlapping spheres, all three with $\varepsilon=0.366$ and (b) the FCC, BCC, and SC packings diluted to $\varepsilon=0.784$. For $\Lambda$, we took 0.25 and smaller to exclude negative error values because for any value of $\Lambda$, the drag error becomes positive with increasing resolution, but for $\Lambda>1$, very fine meshes are necessary to observe this transition. ${ }^{9}$ Figure 4 demonstrates that

- at very low resolutions $\left(d_{\mathrm{sp}}<5\right)$ and low values of $\Lambda$, different packing types can demonstrate geometryspecific error behavior as well as very high convergence rates;

- for each porosity and at moderate or high resolutions, the errors weakly depend on a given geometry and are grouped for a particular value of $\Lambda$; and

- depending on the value of $\Lambda$, the error approaches the -1 convergence rate from below (for $\Lambda=0.25$ or higher) or from above $(\Lambda=1 / 512$ or lower) for both porosities and all geometries.

The last statement extends some of the results of the previous work: ${ }^{9}$ at infinitely high resolution, the convergence rate will be -1.0 for any $\Lambda$ (Fig. 13 in Ref. 9) and, therefore, 


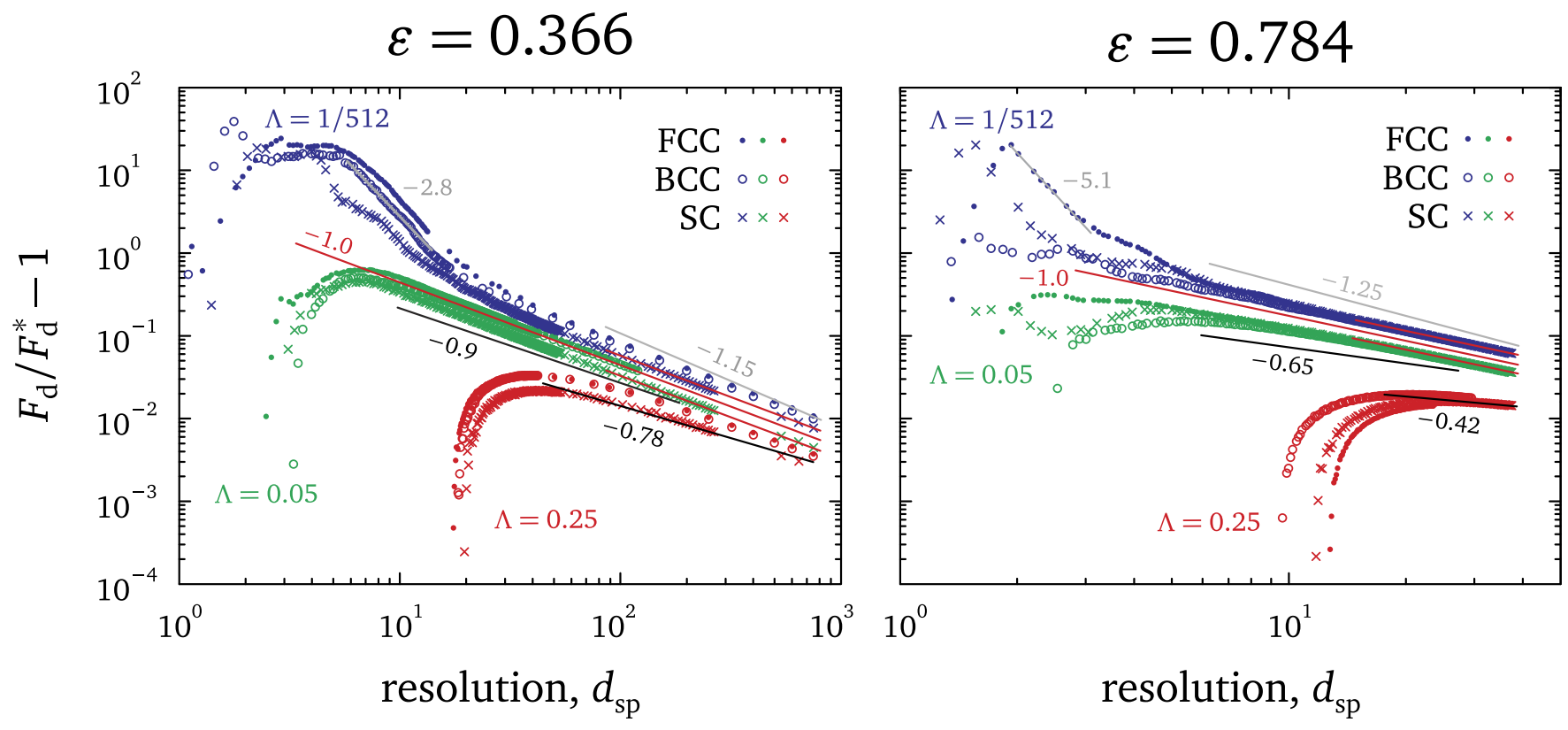

FIG. 4. Relative drag error vs. discretization resolution (in lattice nodes per sphere diameter). FCC, BCC, and SC packings of porosities $\varepsilon=0.366$ and $\varepsilon=0.784$ are considered. The SC packing with $\varepsilon=0.366$ has overlapping spheres while the five other geometries are diluted regular packings. The results are obtained for $\Lambda=1 / 512,0.05,0.25$. The lines indicate various convergence rates: red denotes the -1.0 rate, while gray is faster and black is slower than -1.0 .

extrapolating errors with different values of $\Lambda$ to very high resolution $\left(>10^{4}\right)$ will not allow them to cross each other (Fig. 14 in Ref. 9).

The facts listed above suggest a simple extrapolation approach we discuss in Sec. IV.

\section{EXTRAPOLATION APPROACH}

The convergence rate of the slowest error contribution is -1.0 because of the bounce-back rule. It can be observed for any $\Lambda$ with an increase of resolution. However, the particular choice of $\Lambda$ defines the resolution region, where the error is dominated by the -1.0 contribution. It follows that the "magic parameter" $\Lambda$ can be chosen so as to enforce transition to the -1.0 convergence rate at lower resolutions. After that, any additional increase in resolution leads to linear error behavior and therefore can be predicted using a simple extrapolation. From our experience, the value of $\Lambda$ close to 0.05 leads to the earliest transition to linear convergence for the considered packings and porosities. Therefore, we fix $\Lambda$ to 0.05 for all extrapolation results.

Linear extrapolation requires at least two data points. Because of the residual discrete noise in the drag force values, even after improved discretization, we take six additional points (eight in total) to obtain each extrapolated value. There is no justification for this choice. We simply use additional points to make the extrapolation more robust. However, an important aspect is the distance between maximum $d_{\mathrm{sp}}^{\max }$ and minimum $d_{\mathrm{sp}}^{\min }$ resolutions in this set of points. For example, in the right panel of Fig. 5, one can see oscillations of drag values around the extrapolation line. Such oscillations can impact the extrapolation result if $d_{\mathrm{sp}}^{\max }$ and $d_{\mathrm{sp}}^{\min }$ are too close (e.g., $\left.d_{\mathrm{sp}}^{\min } / d_{\mathrm{sp}}^{\max }<0.95\right)$. Taking $d_{\mathrm{sp}}^{\min }$ too far away from $d_{\mathrm{sp}}^{\max }$ will place $d_{\mathrm{sp}}^{\min }$ in the nonlinear region of the drag curve, see Fig. 4, which too will negatively impact the extrapolation result. We found the ratio $d_{\mathrm{sp}}^{\min } / d_{\mathrm{sp}}^{\max }=0.8$ to be a good choice and use it to collect all extrapolated values. It is worth
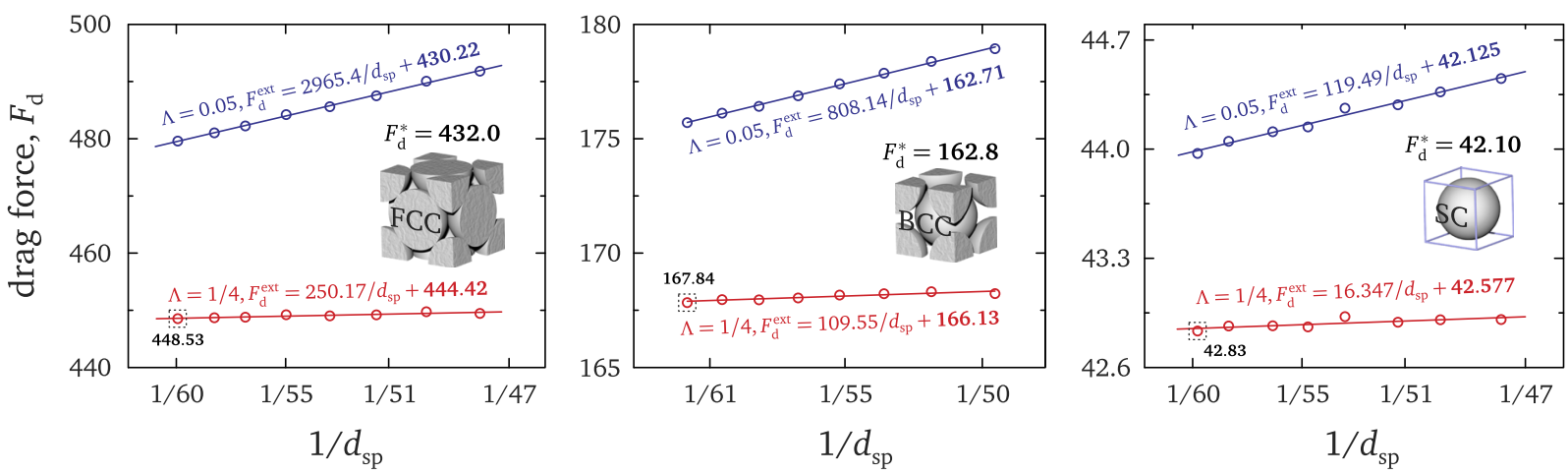

FIG. 5. Examples of extrapolation of drag values toward the infinitely high discretization resolution assuming the solution convergence rate of -1 . The shown results are for the FCC, BCC, and SC packings of touching spheres. All extrapolation results in this study are obtained with $\Lambda=0.05$. 


$\left.\begin{array}{lll}\hline \because:- \text { extrapolation, } \Lambda=0.05 & \circ & \text { BB } \\ \therefore \text { BB this study, } \Lambda=0.25 & \text { ○ } & \text { CLI } \\ \therefore \text { BCLI }\end{array}\right\} \begin{aligned} & \text { [Kh2015], } \\ & \Lambda=3 / 16\end{aligned}$
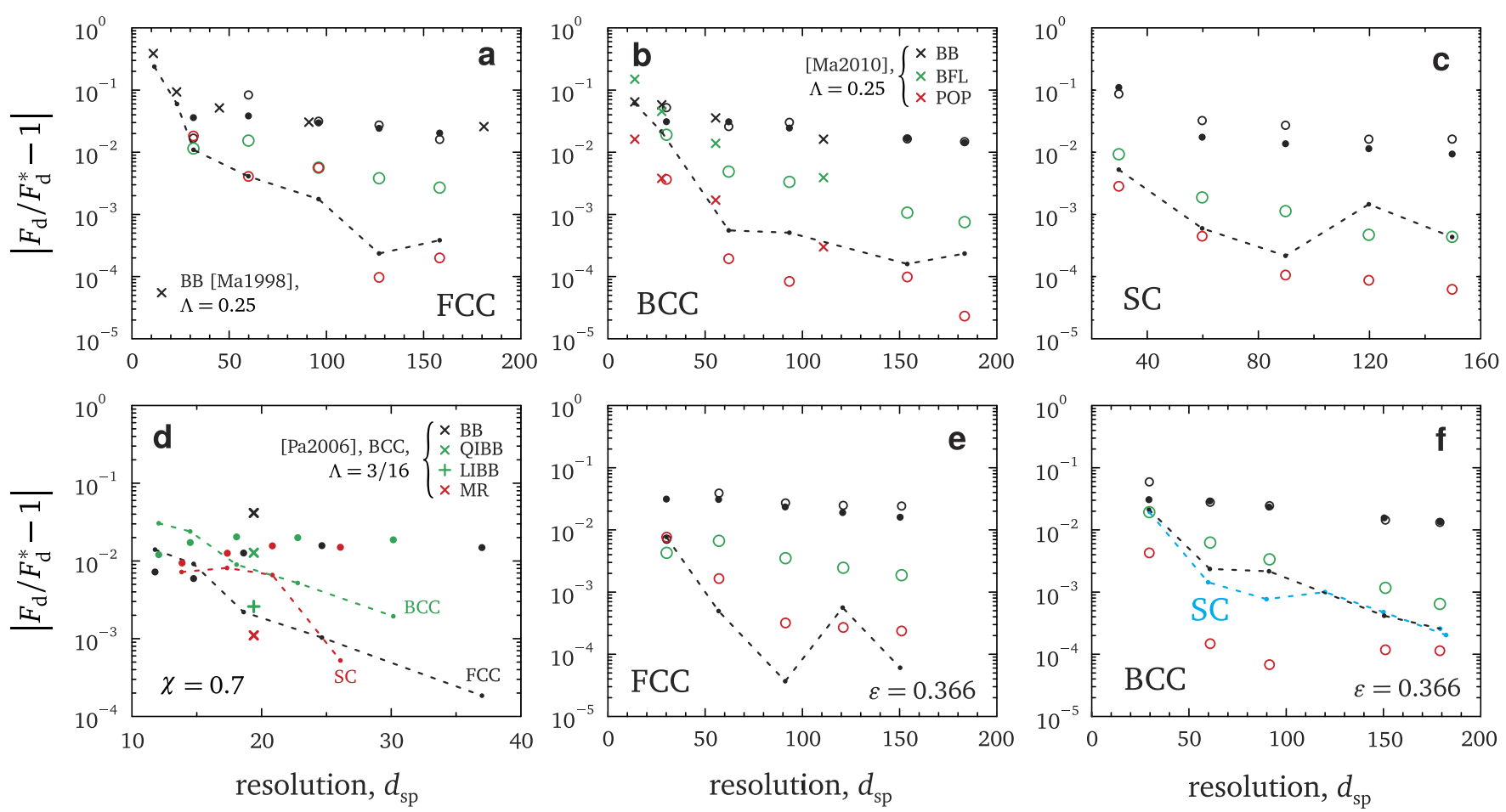

FIG. 6. Relative error in drag force for packings of touching [(a)-(c)] and diluted [(d)-(f)] spheres. [(a)-(c)] Shown are the errors for the extrapolated drag force values (with $d_{\mathrm{sp}}=d_{\mathrm{sp}}^{\max }$ ) and the data taken from other studies: Tables 7, 8, and 10 in the work of Khirevich et al., as well as Table 1 in Ref. 30 and Table II in Ref. 40 in the work of Maier et al. Panel (d) depicts errors for the diluted FCC, BCC, SC arrays with the diameter contraction factor of $\chi=0.7$. The extrapolated values are compared with the results of Pan et al. ${ }^{15}$ for the BCC packing. CLI, MCLI, BFL, POP, QIBB, LIBB, MR refer to the higher-order boundary schemes. [(e) and (f)] Error values for the FCC, BCC, SC packings with $\varepsilon=0.366$.

mentioning that geometries with large amount of irregularly located objects are less impacted by discrete noise, and the statements above are less applicable.

In Fig. 5, we present the extrapolation of drag force described above for the three basic packing types of touching spheres. In Fig. 5, we also show the extrapolation with one of the standard choices of $\Lambda=0.25$ resulting in $\tau=1$ for the BGK model. For instance, a similar approach was discussed in the study of Hoef et al. (Figs. 2 and 3 in Ref. 34), in which the authors assumed quadratic $\left(1 / d_{s p}^{2}\right)$ error convergence. The use of the -2 extrapolation rate for the considered geometries and $\Lambda=0.25$ slightly increases error in estimated drag values (data not shown). Dashed squares highlight the drag values for the highest employed resolutions using the standard simulation approach with $\Lambda=0.25$ without any extrapolation procedures.

To quantify accuracy and to compare the results of our numerical simulations with previous studies, we apply our extrapolation approach to the various packing types and porosities. Accurate reference values are taken from Table I. Figure 6 shows a strong increase of accuracy after applying the extrapolation procedure. The results for the touching spheres of low (FCC, BCC) and moderate (SC) porosities, as well as the porosity of 0.366 , outperform a standard bounce back approach by more than an order of magnitude.
For the high porosities with $\chi=0.7$ that enforces $\varepsilon \approx 0.8$ for all packing types, the extrapolation approach performs comparably to the higher-order boundary schemes reported previously. ${ }^{15}$ We note that in the work of Pan et al., ${ }^{15}$ the authors used two sets of relaxation parameters named "set A" and "set B." We use the data from "set B" that corresponds to the two-relaxation-times collision operator we adopt here; $\tau=0.6$ in their terms corresponds to $\Lambda=3 / 16$ in this study. A slight tuning of $\Lambda$ around 0.05 toward smaller values (see Fig. 4) increases accuracy for the high-porosity case, but our primary focus is on the lowerporosity systems and therefore we stay with a single value of $\Lambda=0.05$.

For the sake of completeness, we note that our attempts to take the large values of $\Lambda$ (say, $\Lambda>10$ ) and apply a similar extrapolation approach to the negative error region did not result in any meaningful outcomes. It appears that the error must cross zero first, become positive, and only then decrease to zero.

\section{v. CONCLUSIONS AND OUTLOOK}

In this paper, we have analyzed the numerical error of the LBM bounce-back approximation of incompressible fluid flow and suggested a simple model for the simulation error. Our approach is based on the existence of at least two error 
contributions with the linear and quadratic decay rates of different signs. These error contributions amplify or compensate each other and, therefore, lead to the very fast $(-3$ or faster) or slow (slower than -0.5 ) transient convergence rates. All of these rates approach the -1 convergence rate sooner or later. This situation is not limited to LBM and also occurs in classic numerical approaches such as the finite difference method-see error behavior in Fig. 3 of Manwart et al. ${ }^{41}$ as an example.

The choice of $\Lambda=0.05$ allowed us to observe the -1 behavior at lower resolutions (of about 20 lattice nodes per sphere diameter for $\varepsilon=0.366$; see Fig. 4) and perform a linear extrapolation of drag values. The extrapolation results reduced the typical LBM/BB error by tens of times at medium resolutions (40 nodes and higher, Fig. 6) and allowed us to achieve accuracy of the high-order CLI and MCLI schemes. We note that there are no difficulties with programming the high-order boundary schemes for simple geometries while developing a serial program implementation of LBM. However, programming complexity increases for parallel applications and when dealing with obstacles distributed irregularly in space (for instance, see Subsection 5.1 in Ref. 30). From this point of view, the suggested extrapolation approach can be seen as an alternative for improving simulation accuracy in some cases without modifying the flow solver.

An extrapolated average velocity value does not provide accurate three-dimensional flow fields, but its knowledge can be used, for example, in simulations of hydrodynamic dispersion. ${ }^{24,44}$ Our preliminary studies suggest that using the extrapolated value of average velocity to calculate the Péclet number improves the accuracy of simulations of hydrodynamic dispersion with a random-walk particle tracking method. ${ }^{24}$ This improvement can be attributed to the fact that the variation of $\Lambda$ moves the location of the zero-velocity boundary in a systematic way within the simulated geometry. A similar improvement can be obtained after scaling the Stokes flow velocity components with their accurate average value obtained from extrapolation.

The approach presented here requires variable resolutions of discretization of the pore space, which is not always possible. Micro-CT images that often provide pore space geometry are usually limited to a single resolution. However, we see this study as a necessary step toward selecting optimal relaxation parameters in the pore-scale lattice-Boltzmann simulations.

\section{ACKNOWLEDGMENTS}

We are grateful for the allocation of computational resources by the Supercomputing Laboratory at King Abdullah University of Science and Technology (KAUST) in Thuwal, Saudi Arabia.

\footnotetext{
${ }^{1}$ B. Ferréol and D. H. Rothman, "Lattice-Boltzmann simulations of flow through Fontainebleau sandstone," Transp. Porous Media 20, 3-20 (1995).

${ }^{2}$ G. Jin, T. W. Patzek, and D. Silin, "SPE 90084-MS: Direct prediction of the absolute permeability of unconsolidated and consolidated reservoir rock," in SPE Annual Technical Conference and Exhibition (SPE, Houston, TX, 2004).
}

${ }^{3}$ T. Ramstad, N. Idowu, C. Nardi, and P.-E. Øren, "Relative permeability calculations from two-phase flow simulations directly on digital images of porous rocks," Transp. Porous Media 94, 487-504 (2012).

${ }^{4}$ G. Jin, T. W. Patzek, and D. B. Silin, "Modeling the impact of rock formation history on the evolution of absolute permeability," J. Pet. Sci. Eng. 100, 153-161 (2012).

${ }^{5}$ D. B. Silin, G. Jin, and T. W. Patzek, "Robust determination of the pore-space morphology in sedimentary rocks," J. Pet. Technol. 56, 69-70 (2004).

${ }^{6}$ D. B. Silin and T. Patzek, "Pore space morphology analysis using maximal inscribed spheres," Physica A 371, 336-360 (2006).

${ }^{7}$ D. Silin, L. Tomutsa, S. M. Benson, and T. W. Patzek, "Microtomography and pore-scale modeling of two-phase fluid distribution," Transp. Porous Media 86, 495-515 (2011).

${ }^{8}$ E. Jettestuen, J. O. Helland, and M. Prodanović, “A level set method for simulating capillary-controlled displacements at the pore scale with nonzero contact angles," Water Resour. Res. 49, 4645-4661 (2013), https:// agupubs.onlinelibrary.wiley.com/doi/pdf/10.1002/wrcr.20334.

${ }^{9}$ S. Khirevich, I. Ginzburg, and U. Tallarek, "Coarse- and fine-grid numerical behavior of MRT/TRT Lattice-Boltzmann schemes in regular and random sphere packings," J. Comput. Phys. 281, 708-742 (2015).

${ }^{10}$ P. L. Bhatnagar, E. P. Gross, and M. Krook, "A model for collision processes in gases. I. Small amplitude processes in charged and neutral one-component systems," Phys. Rev. 22, 511-525 (1954).

${ }^{11}$ Y. Qian, D. D'Humières, and P. Lallemand, "Lattice BGK models for Navier-Stokes equation," Europhys. Lett. 17, 479-484 (1992).

${ }^{12} \mathrm{H}$. Chen, S. Chen, and W. H. Matthaeus, "Recovery of the Navier-Stokes equations using a lattice-gas Boltzmann method," Phys. Rev. A 45, R5339R5342 (1992).

${ }^{13}$ I. Ginzbourg and P. M. Adler, "Boundary flow condition analysis for the three-dimensional lattice Boltzmann model,” J. Phys. II 4, 191-214 (1994).

${ }^{14}$ I. Ginzburg and D. D'Humières, "Multireflection boundary conditions for lattice Boltzmann models," Phys. Rev. E 68, 066614 (2003).

${ }^{15}$ C. Pan, L.-S. Luo, and C. T. Miller, "An evaluation of lattice Boltzmann schemes for porous medium flow simulation," Comput. Fluids 35, 898-909 (2006).

${ }^{16}$ D. D'Humières, “Generalized Lattice-Boltzmann equations," Prog. Astronaut. Aeronaut. 159, 450-458 (1992).

${ }^{17}$ I. Ginzburg, F. Verhaeghe, and D. D'Humières, "Proceedings of eighteenth international symposium on rarefied gas dynamics," Commun. Comput. Phys. 3, 427-478 (2008).

${ }^{18}$ I. Ginzburg, "Equilibrium-type and link-type lattice Boltzmann models for generic advection and anisotropic-dispersion equation," Adv. Water Res. 28, 1171-1195 (2005).

${ }^{19}$ D. D' Humières and I. Ginzburg, "Viscosity independent numerical errors for lattice Boltzmann models: From recurrence equations to 'magic' collision numbers," Comput. Math. Appl. 58, 823-840 (2009).

${ }^{20}$ L. Talon, D. Bauer, N. Gland, S. Youssef, H. Auradou, and I. Ginzburg, "Assessment of the two relaxation time Lattice-Boltzmann scheme to simulate Stokes flow in porous media," Water Resour. Res. 48, W04526 (2012).

${ }^{21}$ A. S. Sangani and A. Acrivos, "Slow flow through a periodic array of spheres," Int. J. Multiphase Flow 8, 343-360 (1982).

${ }^{22}$ A. A. Zick and G. M. Homsy, "Stokes flow through periodic arrays of spheres," J. Fluid Mech. 115, 13-26 (1982).

${ }^{23}$ R. E. Larson and J. J. L. Higdon, "A periodic grain consolidation model of porous media," Phys. Fluids 1, 38-46 (1989).

${ }^{24}$ S. Khirevich, A. Daneyko, A. Höltzel, A. Seidel-Morgenstern, and U. Tallarek, "Statistical analysis of packed beds, the origin of short-range disorder, and its impact on eddy dispersion," J. Chromatogr. A 1217, 4713-4722 (2010).

${ }^{25}$ T. Atmakidis and E. Y. Kenig, "Numerical analysis of residence time distribution in packed bed reactors with irregular particle arrangements," Chem. Prod. Process Model. 10, 17-26 (2015).

${ }^{26} \mathrm{X}$. Frank and P. Perré, "Droplet spreading on a porous surface: A lattice Boltzmann study," Phys. Fluids 24, 042101 (2012).

${ }^{27}$ M. Sbragaglia and S. Succi, "Analytical calculation of slip flow in lattice Boltzmann models with kinetic boundary conditions," Phys. Fluids 17, 093602 (2005).

${ }^{28}$ M. A. A. Spaid and J. F. R. Phelan, "Lattice Boltzmann methods for modeling microscale flow in fibrous porous media," Phys. Fluids 9, 2468-2474 (1997). 
${ }^{29}$ D. Maggiolo, F. Picano, and M. Guarnieri, "Flow and dispersion in anisotropic porous media: A lattice-Boltzmann study," Phys. Fluids 28, 102001 (2016).

${ }^{30}$ R. S. Maier and R. S. Bernard, "Lattice-Boltzmann accuracy in pore-scale flow simulation," J. Comput. Phys. 229, 233-255 (2010).

${ }^{31}$ M. Bouzidi, M. Firdaouss, and P. Lallemand, "Momentum transfer of a Boltzmann-lattice fluid with boundaries," Phys. Fluids 13, 3452-3459 (2001).

${ }^{32}$ M. Junk and Z. Yang, "Convergence of lattice Boltzmann methods for Stokes flows in periodic and bounded domains," Comput. Math. Appl. 55, 1481-1491 (2008).

${ }^{33}$ A. J. C. Ladd, "Numerical simulations of particulate suspensions via a discretized Boltzmann equation. Part 2. Numerical results," J. Fluid Mech. 271, 311-339 (1994).

${ }^{34}$ M. A. van der Hoef, R. Beetstra, and J. A. M. Kuipers, "Lattice-Boltzmann simulations of low-Reynolds-number flow past mono- and bidisperse arrays of spheres: Results for the permeability and drag force," J. Fluid Mech. 528, 233-254 (2005).

${ }^{35}$ Y. Chen, J. R. Third, and C. R. Müller, "A drag force correlation for approximately cubic particles constructed from identical spheres," Chem. Eng. Sci. 123, 146-154 (2015).

${ }^{36}$ B. Chun and A. J. C. Ladd, "Interpolated boundary condition for lattice Boltzmann simulations of flows in narrow gaps," Phys. Rev. E 75, 066705 (2007).
${ }^{37}$ E. Fattahi, C. Waluga, B. Wohlmuth, U. Rüde, M. Manhart, and R. Helmig, "Lattice Boltzmann methods in porous media simulations: From laminar to turbulent flow," Comput. Fluids 140, 247-259 (2016).

${ }^{38}$ G. Silva and V. Semiao, "Consistent lattice Boltzmann modeling of low-speed isothermal flows at finite Knudsen numbers in slip-flow regime: Application to plane boundaries," Phys. Rev. E 96, 013311 (2017).

${ }^{39}$ See http://www.khirevich.com/lbm for S. Khirevich, "MATLAB code for discretization of regular sphere packings;" accessed 15 August 2017.

${ }^{40}$ R. S. Maier, D. M. Kroll, Y. E. Kutsovsky, H. T. Davis, and R. S. Bernard, "Simulation of flow through bead packs using the lattice Boltzmann method," Phys. Fluids 10, 60-74 (1998).

${ }^{41}$ C. Manwart, U. Aaltosalmi, A. Koponen, R. Hilfer, and J. Timonen, "Lattice-Boltzmann and finite-difference simulations for the permeability for three-dimensional porous media," Phys. Rev. E 66, 016702 (2002).

${ }^{42}$ C. Chukwudozie and M. Tyagi, "Pore scale inertial flow simulations in 3-D smooth and rough sphere packs using lattice Boltzmann method," Chem. Eng. Sci. 59, 4858-4870 (2013).

${ }^{43}$ C. J. Roy, "Grid convergence error analysis for mixed-order numerical schemes," AIAA J. 41, 595-604 (2003).

${ }^{44}$ R. S. Maier, D. M. Kroll, R. S. Bernard, S. E. Howington, J. F. Peters, and H. T. Davis, "Pore-scale simulation of dispersion," Phys. Fluids 12, 2065-2079 (2000). 